\title{
Love Waves in a Pre-Stressed Fiber-Reinforced Medium Rest upon a Monoclinic Half-Space
}

\author{
Aditya Kumar Patnaik, Sapan Kumar Samal, Prasanta Kumar Das
}

\begin{abstract}
Love waves in a pre-stressed fiber-reinforced medium lying above a monoclinic half-space have been investigated. Upper surface of fiber reinforced layer remains stress free and interface of half space and layer satisfies continuity conditions .The dispersion equation for Love waves propagation has been derived. Effect of anisotropy parameter of half space and initial stresses of reinforced layer on Love waves propagation have been observed from dispersion curves. Some particular cases have also been developed by using the dispersion equation. Further, the range of the existence of Love waves is calculated. The cut-off periods for three nodes of Love waves with variation of anisotropy parameter and compressive stress are presented in tabular form.

Keywords : Love wave ,Half space, Dispersion, Monoclinic medium.
\end{abstract}

\section{INTRODUCTION}

Civil engineers ,geophysicists and geologists working in reference to internal structure of earth and behavior of earth materials need to have knowledge about the motion of elastic waves in layered media of earth. Ewing et al.[1] illuminates enough information regarding elastic waves in layered media. Chattopadhyay et al.[2] described the properties of SH-waves in a monoclinic crystal medium laid on an irregular half-space. Chattopadhyay et al.[3] discussed the behavior of torsional surface waves propagation in a fiber-reinforced medium. Chattopadhyay et al.[4] studied the behaviour of $\mathrm{SH}$ waves in a monoclinic medium laid between two irregular elastic half-spaces using perturbation techniques. Singh et al.[5] presented a brief discussion on dispersive nature of Love waves in anisotropic monoclinic elastic layers. Other types of layers which are available in the earth are fiber reinforced materials. It is assumed that the formation of reinforced layers are due to high initial stress and energy produced due to tectonic movements of plates inside the earth surface. Some works have also been done recently on fiber reinforced composite materials. Spencer[6] first mentioned the Hooke's law of a fiber-reinforced elastic medium in a preferable direction. Chaudhary et al.[7] made

Revised Manuscript Received on February 05, 2020.

* Correspondence Author

Aditya Kumar Patnaik*, Department of Mathematics, School of Applied Sciences, KIIT, Deemed to be University,, Bhubaneswar-751024, India. Email: pattnaik55@rediffmail.com

Sapan Kumar Samal, Department of Mathematics, School of Applied Sciences,Bhubaneswar-751024, India. Email: sksamal@kiit.ac.in

Prasanta Kumar Das, Department of Mathematics, School of Applied Sciences,KIIT, Deemed to be University, Bhubaneswar-751024,India. Email: pkdasfma@kiit.ac.in

(C) The Authors. Published by Blue Eyes Intelligence Engineering and Sciences Publication (BEIESP). This is an open access article under the CC BY-NC-ND license (http://creativecommons.org/licenses/by-nc-nd/4.0/) an attempt to observe the response of plane SH-waves in an elastic layer lying in between two separated fiber-reinforced materials. Srivastava et al.[8] described the effect of pre-stress in reflection as well as transmission of plane waves in a layered anisotropic media . Gupta et al.[9] threw some light on scattering of plane waves in a fiber-reinforced half-space laid over a triclinic half-space. Ghorai et al[10] observed how the porosity and gravity of medium effective in propagation of Love wave.

In this paper, it has been focussed to investigate the propagation of Love waves in a fiber-reinforced layer of width $\mathrm{H}$ with compressive stress over a monoclinic half space. Taking equations of motion for both fiber rein-forced layer and monoclinic half-space with relevant boundary conditions, frequency equation of Love waves is derived. Phase velocity of Love waves is numerically computed and is presented graphically. Cut-off periods for three nodes of Love waves with varying anisotropy parameter and compressive initial stress are presented in tabular form.

\section{GEOMETRY OF THE MODEL}

A model comprising of a fiber reinforced layer of width $\mathrm{H}$ with initial compressive stress over a monoclinic half space is considered. The contact surface of two medium is at xy-plane and width of upper half layer is $\mathrm{H}$ and $\mathrm{z}$-axis is considered downward normal to the plane of contact. Propagation of Love wave is considered along x-axis, Fig.1.

\section{A. Dynamics of pre-stressed fiber rein-forced medium}

Spencer[5] was first to present the relation between stress and strain for fiber rein-forced elastic material in a preferable direction $\vec{a}$.

$$
\begin{aligned}
\tau_{i j}= & \lambda e_{l l} \delta_{i j}+2 \mu_{T} e_{i j}+\alpha\left(a_{l} a_{m} e_{l m} \delta_{i j}+a_{i} a_{j} e_{l l}\right) \\
& +2\left(\mu_{L}-\mu_{T}\right)\left(a_{i} a_{l} e_{l j}+a_{j} a_{l} e_{l i}\right)+\beta\left(a_{l} a_{m} e_{l m} a_{i} a_{j}\right)
\end{aligned}
$$

Where $\tau_{i j}$ and $e_{i j}=\frac{1}{2}\left(u_{i, j}+u_{j, i}\right)$ denote the stress and strain components,$u_{j}$ be the displacement vector,$\lambda$ is the elastic parameter. $\mu_{L}$ and $\mu_{T}$ are rigidity along longitudinal and transverse directions respectively. Let

$\mathbb{B}=\left\{\vec{b}=\left(b_{1}, b_{2,}, b_{3}\right) \in \mathbb{R}^{3} ;\|\vec{b}\|=1\right.$ where $\left.\|\vec{b}\|^{2}=b_{1}{ }^{2}+b_{2}{ }^{2}+b_{3}{ }^{2}\right\}$

be a directional unit ball in $\mathbb{R}^{3}$ of fiber. 


\section{Love Waves in a Pre-Stressed Fiber-Reinforced Medium Rest upon a Monoclinic Half-Space}

Let $\vec{a} \in \mathbb{B}$ be the direction of the fiber and $\vec{a}=\vec{a}\left(a_{1}, 0, a_{3}\right)$. Propagation of Love waves is along x-axis and the displacement components are taken as $u_{1}=u_{3}=0$ and $u_{2}=u_{2}(x, z, t)$. On Use of these displacement components, the stress components (2.1) are converted to

$$
\begin{gathered}
\tau_{12}=\mu_{T}\left(P \frac{\partial u_{2}}{\partial x}+R \frac{\partial u_{2}}{\partial z}\right), \\
\tau_{23}=\mu_{T}\left(\mathrm{R} \frac{\partial u_{2}}{\partial x}+Q \frac{\partial u_{2}}{\partial z}\right),
\end{gathered}
$$

with

$$
\begin{aligned}
& P=1+\left(\mu_{1}-1\right) a_{1}^{2}, \\
& Q=1+\left(\mu_{1}-1\right) a_{3}^{2}, \\
& R=\left(\mu_{1}-1\right) a_{1} a_{3}, \\
& \text { and } \mu_{1}=\frac{\mu_{L}}{\mu_{T}} .
\end{aligned}
$$

On Consideration of the pre-stress $P_{1}$ along the $X$-axis ,the equation of motion of reinforced layer can be expressed as

$$
\frac{\partial \tau_{12}}{\partial x}+\frac{\partial \tau_{22}}{\partial y}+\frac{\partial \tau_{23}}{\partial z}-P_{1} \frac{\partial \omega_{3}}{\partial x}=\rho \frac{\partial^{2} u_{2}}{\partial t^{2}}
$$

where $\rho$ be the mass per unit volume of the medium and

$\omega_{3}=\frac{1}{2}\left(\frac{\partial u_{2}}{\partial x}\right)$. Using (2.2) and (2.3) in (2.4)

equations become

$$
\left(P-\frac{p_{1}}{2}\right) \frac{\partial^{2} u_{2}}{\partial x^{2}}+2 R \frac{\partial^{2} u_{2}}{\partial x \partial z}+Q \frac{\partial^{2} u_{2}}{\partial z^{2}}=\frac{\rho}{\mu_{T}} \frac{\partial^{2} u_{2}}{\partial t^{2}}
$$

The harmonic solution of $(2.5)$ is taken in the form

$$
u_{2}=\phi(z) e^{i k(x-c t)}
$$

where $k$ is the wave number and $c$ is the phase velocity .

Replacing (2.6) in(2.5) ,it gives

$$
Q \frac{d^{2} \phi}{d z^{2}}+2 \operatorname{rik} \frac{d \phi}{d t}+k^{2}\left(\frac{c^{2}}{c_{T}^{2}}-P+\frac{P_{1}}{2}\right) \phi=0
$$

The solution of above equation is

$$
\phi(z)=A e^{-i k \lambda_{1} z}+B e^{-i k \lambda_{2} z},
$$

Where,

$\lambda_{j}=\frac{1}{Q}\left[R \pm \sqrt{R^{2}+Q\left(\frac{c^{2}}{c_{T}{ }^{2}}-P+\frac{P_{1}}{2}\right)}\right], j=1,2$.

Therefore,

$$
u_{2}=\left(A e^{-i k \lambda_{1} z}+B e^{-i k \lambda_{2} z}\right) e^{-i k(x-c t)}
$$

\section{DYNAMICS OF MONOCLINIC HALF-SPACE}

The monoclinic half-space $(z \geq 0)$ considered is treated as second medium with displacement $v_{2}(x, z, t)$, density $\rho_{2}$ and elastic constants $C_{i j}$. The equation of motion with displacement $v_{2}(x, z, t)$ given by Singh et al[4] is

$c_{66} \frac{\partial^{2} v_{2}}{\partial x^{2}}+2 c_{56} \frac{\partial^{2} v_{2}}{\partial x \partial z}+c_{55} \frac{\partial^{2} v_{2}}{\partial z^{2}}=\rho_{2} \frac{\partial^{2} v_{2}}{\partial t^{2}}$

For propagation of Love wave along $x$-axis with phase velocity $C$, the solution can be taken as

$$
v_{2}=f(z) e^{i k(x-c t)}
$$

Equations(3.1) and (3.2) give

$$
c_{55} f^{\prime \prime}(z)+2 c_{56} i k f^{\prime \prime}(z)-k^{2}\left(c_{66}-\rho_{1} c^{2}\right) f(z)=0
$$

General solution of (3.3) can be expressed as

$$
f(z)=A_{1} e^{i k b_{1} z}+B_{1} e^{-i k b_{2} z}
$$

Where $A_{1}$ and $B_{1}$ are arbitrary constants

$$
\begin{aligned}
& b_{1}=\frac{\sqrt{c_{55}\left(\rho_{2} c^{2}-c_{66}\right)+{c_{56}}^{2}}-c_{56}}{c_{55}}, \\
& b_{2}=\frac{\sqrt{c_{55}\left(\rho_{2} c^{2}-c_{66}\right)+{c_{56}}^{2}}+c_{56}}{c_{55}}
\end{aligned}
$$

Equations (3.2) and (3.4) yield

$$
v_{2}=\left(A_{1} e^{i k b_{1} z}+B_{1} e^{-i k b_{2} z}\right) e^{i k(x-c t)}
$$

For the Love wave $v_{2} \rightarrow 0$ as $Z \rightarrow \infty$

The solution (3.5) can be expressed as

$$
v_{2}=\left(B_{1} e^{-i k b_{2} z}\right) e^{i k(x-c t)}
$$

\section{BOUNDARY CONDITIONS AND SOLUTION}

The relevant conditions for boundary are taken as

$$
\begin{aligned}
& \tau_{23}=0 \text { at } \mathrm{z}=-H \\
& u_{2}=v_{2} \text { at } \mathrm{z}=0 \\
& \tau_{23}=\sigma_{23} \text { at } \mathrm{z}=0
\end{aligned}
$$

Where,

$$
\tau_{23}=\mu_{T}\left(R \frac{\partial u_{2}}{\partial x}+Q \frac{\partial u_{2}}{\partial z}\right) ।
$$




$$
\sigma_{23}=c_{55} \frac{\partial v_{2}}{\partial z}+c_{56} \frac{\partial v_{2}}{\partial x}
$$

On use of (4.3) in boundary conditions (4.1) and(4.2), it yields following equations

$$
\left(R-Q \lambda_{1}\right) e^{i k m_{1} H} A+\left(R-Q \lambda_{2}\right) e^{i k m_{2} H} B=0
$$

$$
\begin{gathered}
\left(R-Q \lambda_{1}\right) A+\mu_{T}\left(R-Q \lambda_{2}\right) B=c_{55} b_{1}+c_{66} \\
A+B=A_{1}
\end{gathered}
$$

On elimination of the constants from(4.4) to (4.6) , the dispersion equation can be expressed as

$$
\begin{array}{r}
\tan \left(\sqrt{\frac{R^{2}}{Q^{2}}+\frac{1}{Q}\left(\frac{c^{2}}{c_{T}{ }^{2}}-P+\frac{P_{1}}{2}\right)}\right) k H= \\
\frac{\left(c_{55} c_{66}\right)^{\frac{1}{2}} \sqrt{1-\frac{c^{2}}{\alpha_{1}{ }^{2}}-\varepsilon}}{\mu_{T} \sqrt{R^{2}+Q \sqrt{\frac{c^{2}}{c_{T}{ }^{2}}-P+\frac{P_{1}}{2}}}}
\end{array}
$$

Where

$$
\varepsilon=\frac{c_{56}{ }^{2}}{c_{55} c_{66}}, \alpha_{1}^{2}=\frac{c_{66}}{\rho_{2}} .
$$

Equation (4.7) is the dispersion equation of Love waves in a pre-stressed fiber rein-forced layer of width $H$ laid above a monoclinic half-space.

\section{RANGE OF VELOCITY}

Range of phase velocity of Love waves has important role in theoretical and practical point of view in earth science and is helpful to analyze the (4.7) .From (4.7), it can be observed that Love wave can propagate in a pre-stressed fiber rein-forced layer medium over a monoclinic half-space when

$$
c_{T} \sqrt{P-\frac{P_{1}}{2}-\frac{R^{2}}{Q}}<c<\alpha_{1} \sqrt{1-\varepsilon}
$$

The equation(5.1) describes the significance of initial stress and anisotropy parameter of the medium for existence of phase velocity. The dispersion equation of Love waves in a pre-stressed fiber reinforced plate of width $H$ is converted to

$$
\tan \left(\sqrt{\frac{R^{2}}{Q^{2}}+\frac{1}{Q}\left(\frac{c^{2}}{c_{T}{ }^{2}}-P+\frac{P_{1}}{2}\right)}\right) k H=0 \text { and this }
$$

implies $C>C_{T} \sqrt{P-\frac{P_{1}}{2}-\frac{R^{2}}{Q}}$ and
$\left(\sqrt{\frac{R^{2}}{Q^{2}}+\frac{1}{Q}\left(\frac{c^{2}}{c_{T}^{2}}-P+\frac{P_{1}}{2}\right)}\right) k H=n \pi, n=0,1,2,3$

This equation also holds for an initialiy stressed fiber reinforced layer with fluid layers on both sides

\section{PARTICULAR CASES}

\section{A. Case-1: Orthotropic Medium}

For orthotropic medium $c_{56}=0$ and taking $\varepsilon=0$ and taking $\mu_{L}=\mu_{T}=1, P_{1}=0, P=1, \mathrm{Q}=1, \mathrm{R}=0 \quad$ for reinforced layer, the dispersion equation $(4.7)$ is converted to

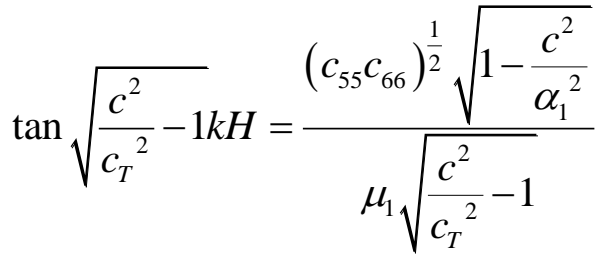

Dispersion equation(5.2) of Love waves in an orthotropic plate of width $H$ becomes

$$
\sqrt{\frac{c^{2}}{c_{T}^{2}}-1 k H}=n \pi, n=0,1,2
$$

\section{B. Case-2: Transversely isotropic medium}

Equations (6.1) and (6.2) are also well agreed with when both the media are transversely isotropic.

\section{Case-3: Isotropic medium}

For isotropic medium following constants can be taken as

$$
\begin{aligned}
& c_{55}=c_{66}=\mu_{2}^{\prime} \\
& \mu_{L}=\mu_{T}=\mu_{1}^{\prime} \\
& c_{56}=0, \varepsilon=0
\end{aligned}
$$

Use of these relations in the frequency equation (4.7) gives

$$
\tan \left(\sqrt{\frac{c^{2}}{c_{T}^{2}}-1}\right) k H=\frac{\mu_{2}^{\prime} \sqrt{1-\frac{c^{2}}{\alpha_{1}^{2}}}}{\mu_{1}^{\prime} \sqrt{\frac{c^{2}}{c_{T}{ }^{2}}-1}}
$$

This is in well agreement with standard frequency equation of isotropic media. 


\section{Love Waves in a Pre-Stressed Fiber-Reinforced Medium Rest upon a Monoclinic Half-Space}

\section{Case4: Free initial stress in upper medium}

When no initial stress is there in upper layer ,i.e., when $P_{1}=0$, then frequency equation can be expressed as $\tan \left(\sqrt{\frac{R^{2}}{Q^{2}}+\frac{1}{Q}\left(\frac{c^{2}}{c_{T}{ }^{2}}-1\right)}\right) k H=\frac{\left(c_{55} c_{66}\right)^{\frac{1}{2}} \sqrt{1-\frac{c^{2}}{\alpha_{1}{ }^{2}}-\varepsilon}}{\mu_{T} \sqrt{R^{2}+Q\left(\frac{c^{2}}{c_{T}{ }^{2}}\right)-P}}$

\section{NUMERICAL CALCULATIONS}

For numerical calculation, It is assumed that

$$
\begin{aligned}
& c_{55}=c_{66} \\
& \frac{\alpha_{1}}{c_{T}}=b \\
& \frac{c_{55}}{\mu_{T}}=a
\end{aligned}
$$

Equation (4.7) can be expressed as

$$
\begin{aligned}
& \tan \left(\sqrt{\frac{R^{2}}{Q^{2}}+\frac{1}{Q}\left(\frac{c^{2}}{c_{T}{ }^{2}}-P+\frac{P_{1}}{2}\right)}\right) k H= \\
& \frac{a \sqrt{1-\frac{c^{2}}{b^{2} c_{T}^{2}}-\varepsilon}}{\sqrt{R^{2}+Q \sqrt{\frac{c^{2}}{c_{T}^{2}}-P+\frac{P_{1}}{2}}}} \\
& \text { and } \sqrt{P-\frac{P_{1}}{2}-\frac{R^{2}}{Q}}<\frac{c}{c_{T}}<b(1-\varepsilon)^{\frac{1}{2}}
\end{aligned}
$$

Dispersion curves drawn from(7.2) depict the change of phase velocity with the wave numbers .Fig.2 is plotted by taking $a=2, b=1.3$ and upper layer isotropic and free from initial stress i.e. $\mu_{1}=1, P_{1}=0$ for various values of the anisotropic parameter $\varepsilon$ of lower half space. $\varepsilon=0$ indicates the lower medium isotropic. It is found that the decrease in phase velocity results increase in anisotropic parameter $\varepsilon$ and wave number.Fig.3 is drawn by taking $\quad a_{1}=\frac{\sqrt{3}}{2}, a_{3}=\frac{1}{2}, \mu_{1}=\frac{2.56}{2.54}, P_{1}=0 \quad$ for compressive stress free upper layer and for various anisotropic parameters for lower half-space .It is seen that the phase velocity is inversely proportional to wave number.

Fig.4,Fig.5 describe the change in phase velocity with non-dimensional wave number for $P_{1}=0.1$ and $P_{1}=0.2$ respectively.Fig. 6 shows the dispersion curves for varying stresses in the fiber rein-forced medium laid above anisotropic half space .It is observed that the phase velocity and stresses are inversely proportional to each other. From condition (7.3) we see that the presence of anisotropy parameter $\varepsilon$ reduces the range of phase velocity and the range is given by

$$
c_{T} \sqrt{P-\frac{P_{1}}{2}-\frac{R^{2}}{Q}}<c<\alpha_{1}(1-\varepsilon)^{\frac{1}{2}}
$$

From (4.7), One can get the cutoff period for the $n^{\text {th }}$ nodes and is given by

$$
T_{n}=\frac{2 H}{n c_{T} Q} \sqrt{1-\left(\frac{P-\frac{R^{2}}{Q}-\frac{P_{1}}{2}}{1-\varepsilon}\right)\left(\frac{c_{T}}{\alpha_{1}}\right)^{2}}
$$

To know the influence of initial stress on the cutoff period ,following assumptions are considered .For isotropic half space

$\varepsilon=0, H=35 \mathrm{~km}, c_{T}=3.5 \mathrm{~km} / \mathrm{sec}, \alpha_{1}=4.5 \mathrm{~km} / \mathrm{sec}$

Table-I: cutoff period for three nodes $(n=1,2,3)$ for $\varepsilon=0$ and various values of $P_{1}$ of layer

\begin{tabular}{|l|l|l|l|l|}
\hline $\mathbf{N}$ & isotropic & \multicolumn{3}{|c|}{ non isotropic $\mu_{1}=\frac{2.56}{2.54}$} \\
& & \multicolumn{3}{|l|}{} \\
\hline & $\mu_{1}=1, \varepsilon=0$ & $P_{1}=0.2$ & $P_{1}=0.4$ & $P_{1}=0.6$ \\
\hline 1 & 12.57 & 13.0026 & 13.9689 & 14.7931 \\
\hline 2 & 6.28539 & 6.54632 & 6.98446 & 7.39662 \\
\hline 3 & 4.19026 & 4.36421 & 4.6563 & 1.93112 \\
\hline
\end{tabular}

Table-II: cutoff period for three nodes for $P_{1}=0$ and various values of $\varepsilon$

\begin{tabular}{|c|c|c|c|c|}
\hline & isotropic & non isotropic & $\mu_{1}=\frac{2.56}{2.54}, P_{1}=0$ \\
n & & & \\
\hline & $\mu_{1}=1, \varepsilon=0$ & $\varepsilon=0.1$ & $\varepsilon=0.2$ & $\varepsilon=0.3$ \\
\hline 1 & 12.57 & 10.98 & 9.30764 & 7.55816 \\
\hline 2 & 6.28539 & 5.49002 & 4.6532 & 3.27908 \\
\hline 3 & 4.19026 & 3.66001 & 3.10255 & 2.18605 \\
\hline
\end{tabular}

Table-III: cutoff period for first three nodes for $\boldsymbol{P}_{\mathbf{1}}=\mathbf{0 . 2}$ and various values of $\varepsilon$

\begin{tabular}{|c|c|c|c|c|}
\hline n & isotropic & \multicolumn{3}{|c|}{ non isotropic $\mu_{1}=\frac{2.56}{2.54}, P_{1}=0.2$} \\
\hline & $\begin{array}{c}\mu_{1}=1, \\
\varepsilon=0\end{array}$ & $\varepsilon=0.1$ & $\varepsilon=0.2$ & $\varepsilon=0.3$ \\
\hline 1 & 12.57 & 12.1206 & 10.783 & 8.76842 \\
\hline 2 & 6.28539 & 6.0603 & 5.39151 & 4.38421 \\
\hline 3 & 4.19026 & 4.0402 & 3.59434 & 2.92281 \\
\hline
\end{tabular}

Tables-I,II,III give the values of cutoff period for three nodes for various values of initial stress $P_{1}$ keeping anisotropy 
parameter $\varepsilon=0$ and for various values of anisotropy parameter $\varepsilon$ keeping initial stress $p_{1}=0$ and 0.2 .It is observed from table-I that the cutoff period increases as the initial stress in the layer medium increases .Table-II shows that the cutoff period of Love wave decreases as the value

of anisotropy parameter of the half-space increases .Table-III shows that the cutoff periods also decreases as the anisotropy parameter of the half-space increases for a fixed initial stress $P_{1}=0.2$.

\section{CONCLUSION}

The cutoff period of first three nodes increases significantly due to increase in the initial stress of the half-space and decreases due to in the anisotropy parameter $\varepsilon$.It is observed that the influence of anisotropy simultaneously in Love wave propagation is significant. It is found that increase in anisotropy of half-space results diminish in phase velocity of Love waves and the magnitude number.

The phase velocity of rein-forced layer in the absence of half-space increases. It is also concluded that phase velocity has decreasing tendency with the increment of the initial stress in layer. Finally it is concluded that the energy propagation in the form of velocity of Love waves in a self reinforced layer in the presence of initial stress and anisotropic parameter of half space is very less in comparison to stress free and isotropic medium

\section{REFERENCES}

1. M. Ewing, W. Jardetzky and F.Press, Elastic waves in layered media 1957.

2. A.Chattopadhyay, S.Gupta, V.K.Sharma and P.Kumari," Propagation of SH waves in an irregular monoclinic crustal layer,"Applied Mechanics., 2008,78(12), pp. 989-999,Applied mathematical sciences 4(44) 2157-2170.

3. A. Chattopadhyay, S.Gupta, S.K.Samal,and V.K.Sharma,Torsional wave in self-reinforced medium, 2009.

4. A.Chattopadhyay, S.Gupta,A.K.Singh and S.A.Sahu "Propagation of over a semi-infinite monoclinic medium",Int.Journal of geomechanics ,9(1),2010,pp.9-13.

5. S.Singh,N.Sachdeva and S.Khurana, "A note on the dispersionof Love waves in a Layered monoclinic elastic media",Proc.Indian Acad. Sci.(Math.Sci.),Vol.109,(4),1999,pp. 417-423.

6. A.J.M.Spencer, "Deformation of fiber reinforced material," oxford University Press.,London,1972..

7. S.Chaudhary,V.P.Kauhsik and S.K.Tomar,'Plane SH-Wave Response from Elastic Slab interposed between two different Self Reinforced Elastic Solids,"Int.Journal of App.Mechanics and Engineering, McGraw-Hill,New York, 11(4) ,2006,pp. 787-801.

8. A.Srivastav,A.Chattopadhyay,P.Sing,A.K.Singh,'Two-Dimensional Plane Wave Reflection and Transmission in a Layered Highly Anisotropic Media under Intial Stress," Journal of Earthquake Engineering, Taylor and Francis,2018,pp. 1-19.

9. S.Gupta,A.Pramanik,Smita and S.Pramanik," Scattering of three dimensional plane waves in a self-reinforced half-space lying over a triclinic half-space," Journal of Geophysics and Engineering,15(3),2018,75.

10. Ghorai,P.Anjana,S.K.Samal,N.C.Mohanti, "Love waves in a elastic half-space under gravity,"Applied Mathematical modeling,34,2010,pp.1873-1883. parameter of the half space and initial stress of the layer of phase velocity is inversely proportional to the wave initial stress decreases as the anisotropy parameter of $\mathrm{SH}$ waves in an irregular non homogeneous monoclinic crustal layer fluid-saturated porous layer under a rigid boundary and lying over an

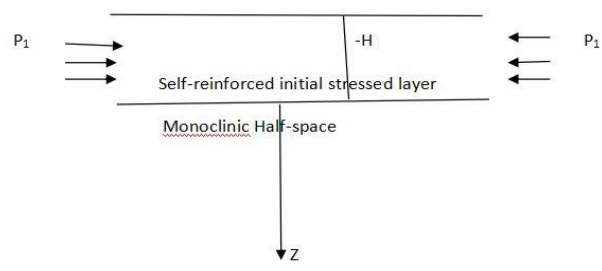

Fig.1.Geometry of the problem

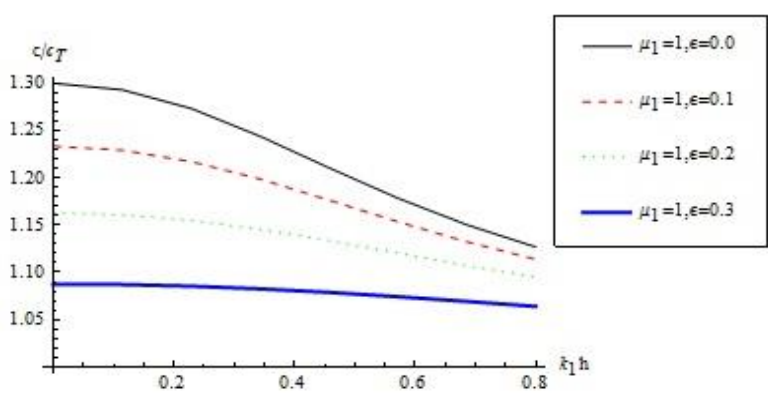

Fig.2.Variation of phase velocity with non dimensional wave number for the fundamental Love mode for various values of anisotropic parameter $\varepsilon$ of half-space with fixed upper layer isotropic $\mu_{1}=1$ and initial stress $p_{1}=0$
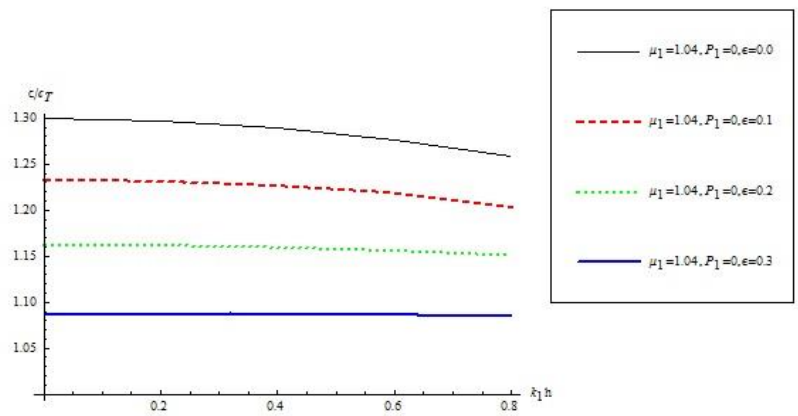

Fig.3.Variation of phase velocity with non dimensional wave number for the fundamental Love mode for various values of anisotropic parameters $\varepsilon$ of half-space with fixed upper layer isotropic $\mu_{1}=1.04$ and initial stress $P_{1}=0$

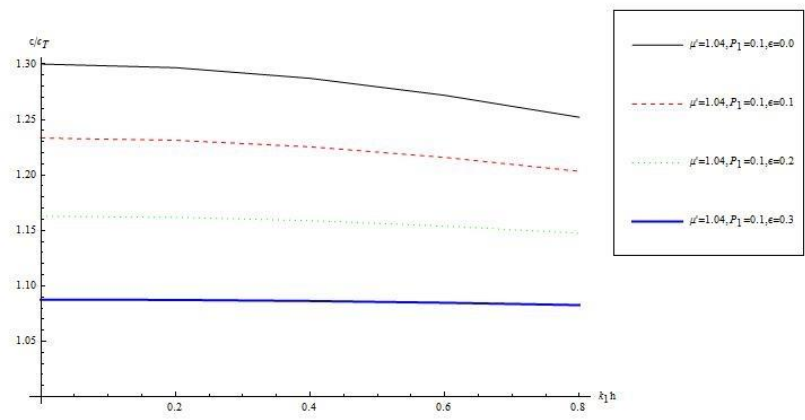

Fig.4.Variation of phase velocity with dimensional wave number for the fundamental Love mode for various values of anisotropic parameters $\varepsilon$ of half-space with fixed $\mu_{1}=1.04$ and initial stress $P_{1}=0.1$ for upper layer 

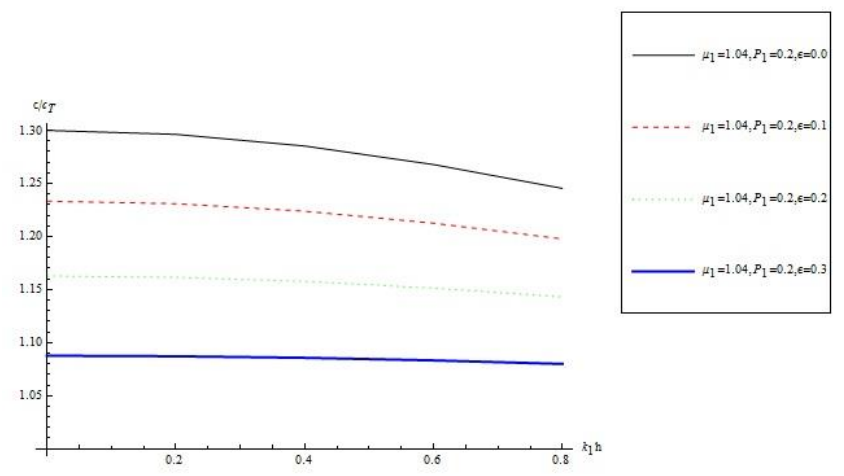

Fig.5.Variation of phase velocity with no dimensional wave number for the fundamental Love mode for various values of anisotropic parameters $\varepsilon$ of half-space with

fixed $\mu_{1}=1.04$ and initial stress $P_{1}=0.2$ for upper layer
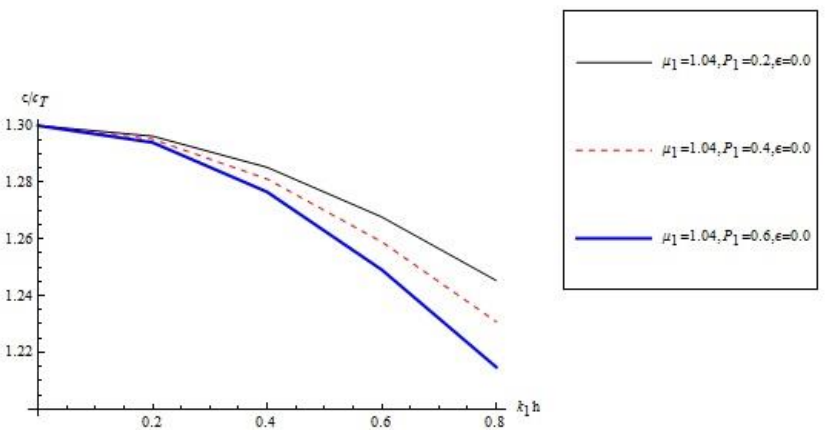

Fig.6.Variation of phase velocity with non-dimensional wave number for the fundamental Love mode for various

values of initial stress $P_{1}$ of layer with fixed $\mu_{1}=1.04$

$$
\text { and } \varepsilon=0.0
$$

\section{AUTHORS PROFILE}

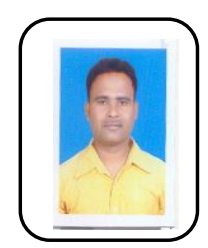

Aditya Kumar Ptnaik, is Research Scholar of Mathematics in School of Applied Sciences, KIIT Deemed to be University Bhubaneswar. His research area is differential equation and Theoretical Seismology

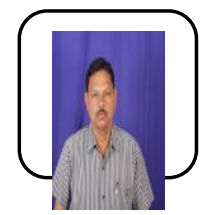

Dr. Sapan Kumar Samal, is professor of Mathematics in School of Applied Sciences, KIIT, Deemed to be University.His research area is differential equation, Numerical Analysis and Theoretical Seismology.He guided four students for Ph.D. Program and Two students are continuing

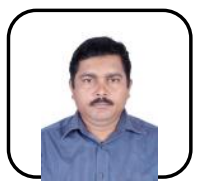

Dr. P.K.Das, is Associate Professor of Mathematics in KIIT Deemed to be University Bhubaneswar. He has 14 years of teaching experience as well as 16 years of research experience. He has supervised 6 Ph.D. scholars. He has published 49 peer reviewed international journals (Scopus indexed, WoS, UGC approved, and SCI). His area of research works are

1. Theory of variational inequalities

2. Theory of complementarity problems

3. Fixed point theory and projection methods

4. Numerical methods of variational inequality problems

5. Convex optimization

6. Invex optimization

7. Summability theory

8. Operator theory

9. Differential equations 